\title{
Livre blanc : Une approche moderne à la réalité d'aujourd'hui
}

\author{
Michel Paradis, B. Sc. LL.B. \\ Conseiller en stratégie - MJP Services Conseils
}

\section{INTRODUCTION}

\section{Depuis plusieurs années, nous avons été témoins de changements importants de notre environnement socioéconomique. Il est devenu incontestable, avec le temps, que pour bien des communautés, la \\ municipalité est devenue un acteur majeur du développement économique de nombreuses collectivités.}

Lors du congrès de l'UMQ qui s'est tenu en mai 2012, une étape importante a été franchie dans la réflexion relative à ce nécessaire repositionnement du rôle du palier de gouvernement municipal. Considérant que notre champ d'intérêt concerne le développement économique, la lecture des thèmes contenus dans ce document de réflexion ne pouvait que susciter notre intérêt. Un constat s'impose: il s'agit d'une démarche nécessaire pour que nos instances municipales puissent pleinement jouer leur rôle d'acteurs de premier plan au développement économique de nos communautés.

Depuis plusieurs années, nous avons été témoins de changements importants de notre environnement socioéconomique. Il est devenu incontestable, avec le temps, que pour bien des communautés, la municipalité est devenue un acteur majeur du développement économique de nombreuses collectivités. Conséquence de cette réalité qui a pris forme, de nombreuses responsabilités additionnelles incombent maintenant à

\section{BRÈVE MISE EN CONTEXTE}

Une première réalité qui peut nous sembler surprenante est que même si nous vivons dans un contexte nord-américain qui, au plan économique, tend vers une ouverture toujours plus grande de nos frontières et à une harmonisation au plan réglementaire, la comparaison n'est pas applicable en ce qui concerne nos municipalités nos instances municipales. Il n'est donc pas surprenant que les leaders municipaux du Québec aient décidé d'entreprendre cette réflexion sur la gouvernance de nos municipalités, notamment sous l'angle d'une révision des pouvoirs réels que celles-ci possèdent pour assumer les responsabilités dont elles ont la charge.

Les municipalités canadiennes et québécoises ont été particulièrement parties prenantes d'initiatives de relances notamment en ce qui concerne nos infrastructures et le seront encore. Possiblement plus fortement que jamais au cours de cette période, les instances municipales ont été des partenaires névralgiques pour atténuer les impacts potentiels d'un ralentissement économique appréhendé. Une des conclusions de cette période est fort probablement que dans une économie ouverte et mondialisée comme la nôtre, le palier de gouvernement municipal est devenu sans aucun doute un levier du développement économique essentiel, pour ceux et celles qui en doutaient encore, de l'ensemble des régions québécoises et canadiennes.

Les municipalités canadiennes et québécoises ont été particulièrement parties prenantes d'initiatives de relances notamment en ce qui concerne nos infrastructures et le seront encore. 
aussi bien outillées que les villes américaines. Malheureusement, ce n'est pas le cas.

Force est de souhaiter que la démarche enclenchée par le Livre blanc permettra de corriger le tir. Par exemple, lorsque le document de consultation du Livre blanc souligne qu'une des faiblesses est l'existence d'une fiscalité inadaptée à la mission sociale et économique d'aujourd'hui en précisant que l'impôt foncier est la principale source de financement des municipalités, il nous apparait que cette situation est plus que symptomatique. En effet, déjà la Fédération canadienne des municipalités mettait en lumière certaines incohérences ${ }^{1}$ en 2001 tout comme dans sa plus récente publication sur l'état des villes et des collectivités du Canada ${ }^{2}$.

Ainsi, on constate que les municipalités américaines œuvrent, dans l'ensemble, dans un cadre fiscal plus permissif qu'au Québec ou au Canada. En effet, de 2001 à 2008, près de $50 \%$ des dépenses effectuées par nos municipalités canadiennes et québécoises étaient soutenues par des revenus provenant de l'impôt foncier. Cette part ne représentait que $23 \%$ des sources de revenus pour l'ensemble des municipalités américaines $^{3}$ et plus de $30 \%$ de leurs autres sources de revenus provenaient, par exemple, de frais d'utilisation alors que pour nos municipalités canadiennes et québécoises, on estime cette part à environ $20 \%$.

\section{On constate que les municipalités américaines œuvrent, dans l'ensemble, dans un cadre fiscal plus permissif qu'au Québec ou au Canada.}

On se rend vite compte qu'une telle situation doit être perçue comme une difficulté importante si l'enjeu est regardé dans une perspective municipale. Bref, on peut se poser la question suivante : y a-t-il des raisons factuelles au plan du développement économique qui peut expliquer cette impression de difficulté visant à accorder aux municipalités une réelle transformation afin de leur permettre d'être plus autonomes?

\section{DÉCENTRALISATION ET AUTONOMIE LOCALE : DES RÉSULTATS ÉCONOMIQUES PORTEURS}

À ce questionnement, nous pouvons répondre que des initiatives ont été mises en œuvre au Québec. Celle que nous pouvons citer et qui visa à accorder une plus grande capacité d'intervention des acteurs municipaux fut la Politique nationale de la ruralité adoptée en 2001 et renouvelée en 2006. En effet, par le caractère décentralisé de ce programme, les acteurs locaux pouvaient mener diverses démarches en matière de développement économique.

Une analyse plus que pertinente de résultats obtenus par ce programme fût réalisée et supervisée par André Joyal, professeur et chercheur à l'UQTR au sein de l'Institut de recherche sur les PME, spécialiste du développement local et régional $^{4,5}$. Ainsi, dans les MRC qui furent observées, on constate que les sommes accordées aux MRC par le biais du programme du Pacte rural et utilisé afin de favoriser le développement de projets ont été des effets de levier majeur. En effet, on souligna la création de partenariats avec des organismes de dévelop- pements, d'institutions financières et d'autres paliers de gouvernements. Ces projets suscitèrent particulièrement une crédibilité, car ils étaient issus de la collectivité.

Le rôle du maire était très souvent un facteur-clé pour assumer une direction dans la réalisation de ces projets auxquels le Pacte rural était un des outils utilisés et que la souplesse du programme permit aux acteurs locaux, dont municipaux, de pouvoir partager des pistes de solutions face à certains enjeux économiques, avec la population.

On remarqua que le rôle du maire était très souvent un facteur-clé pour assumer une direction dans la réalisation de ces projets auxquels le Pacte rural était un des outils utilisés et que la souplesse du programme permit aux acteurs locaux, dont municipaux, de pouvoir partager 
des pistes de solutions face à certains enjeux économiques, avec la population. Bref, les résultats démontrent que les actions visant une plus grande autonomie de nos instances locales permettant un rôle plus significatif de nos élus locaux et régionaux s'avèrent être importantes dans une perspective de développement économique. Y a-t-il une surprise à ce constat quand on sait qu'ailleurs, hors du Québec et du Canada, que les intervenants du monde municipal ont déjà des moyens d'interventions plus élaborés ou encore une autonomie plus importante? Poser la question c'est y répondre et un effet certain de ces recherches renforce la nécessité de la démarche entreprise par l'UMQ et le Livre blanc qui en découle.

\section{NOUVEAU PACTE FISCAL : SUITE LOGIQUE AU LIVRE BLANC?}

Le 27 novembre 2012, nous pouvions prendre connaissance par le biais des médias que la question d'un nouveau pacte fiscal a été abordée en la présence du nouveau ministre des Affaires municipales et de la Table québécoise des municipalités. Des mots tels qu'« autonomie municipale » et «décentralisation» font partie des vocables que nous pouvons actuellement entendre.

Il importe plus que jamais de profiter de cette occasion, non seulement de remettre en question la situation actuelle des moyens accordés aux villes du Québec d'accroître leur autonomie en matière de sources de financement, mais aussi d'en accepter la diversité. En ce sens, l'exemple américain peut être fort inspirant ${ }^{6}$. Bien qu'en revanche, ceci génère une plus grande part de responsabilité des leaders municipaux, force est d'admettre que nous en sommes maintenant à ce besoin d'un rôle plus important de nos municipalités comme acteurs du développement économique :

The action-reactive and proactive-as well as the rhetoric of state and local elected and appointed officials have significant and direct (as well as indirect) impacts on the ability of the private sector to create employment opportunities and wealth at the local and regional levels.

(Gerald L. Gordon, 2012, p.2)

Cette démarche d'un nouveau pacte fiscal devrait tendre, souhaitons-le, à nous projeter vers une modernité qui tient compte du contexte économique global d'aujourd'hui et l'initiative mise de l'avant par l'UMQ et son Livre blanc est assurément contributif à cette heureuse tendance que prend cet enjeu.

\section{BIBLIOGRAPHIE ET NOTES}

${ }^{1}$ Fédération canadienne des municipalités, RésuméAlerte, les villes canadiennes seront-elles en mesure de concurrencer? Un survol comparatif des gouvernements municipaux au Canada, aux États-Unis et en Europe, Ottawa, mai 2001, pp. 2-3.

${ }^{2}$ Fédération canadienne des municipalités, L'état des villes et des collectivités du Canada 2012, Ottawa, 2012, p.5

${ }^{3}$ Fédération canadienne des municipalités, Énoncé de politique sur les Finances municipales et les accords intergouvernementaux, mars 2012, p.1

${ }^{4}$ Joyal A. et K. ElBatal, 2008 « La gouvernance rurale vue à travers la politique nationale de la ruralité » Canadian Journal of Regional Science/Revue canadienne des sciences régionales, XXXI : 3 (Autumn/automne 2008), 601-615.

${ }^{5}$ Joyal A. et K. El Batal, 2007 «Décentralisation et développement rural. Étude de cas québécois » Revue d'Économie Régionale \& Urbaine 2, 251-267.

${ }^{6}$ Gordon G.L. (2012). Reinventing Local and Regional Economics, Boca Raton (Fl), "CRC Press", 339 p. 


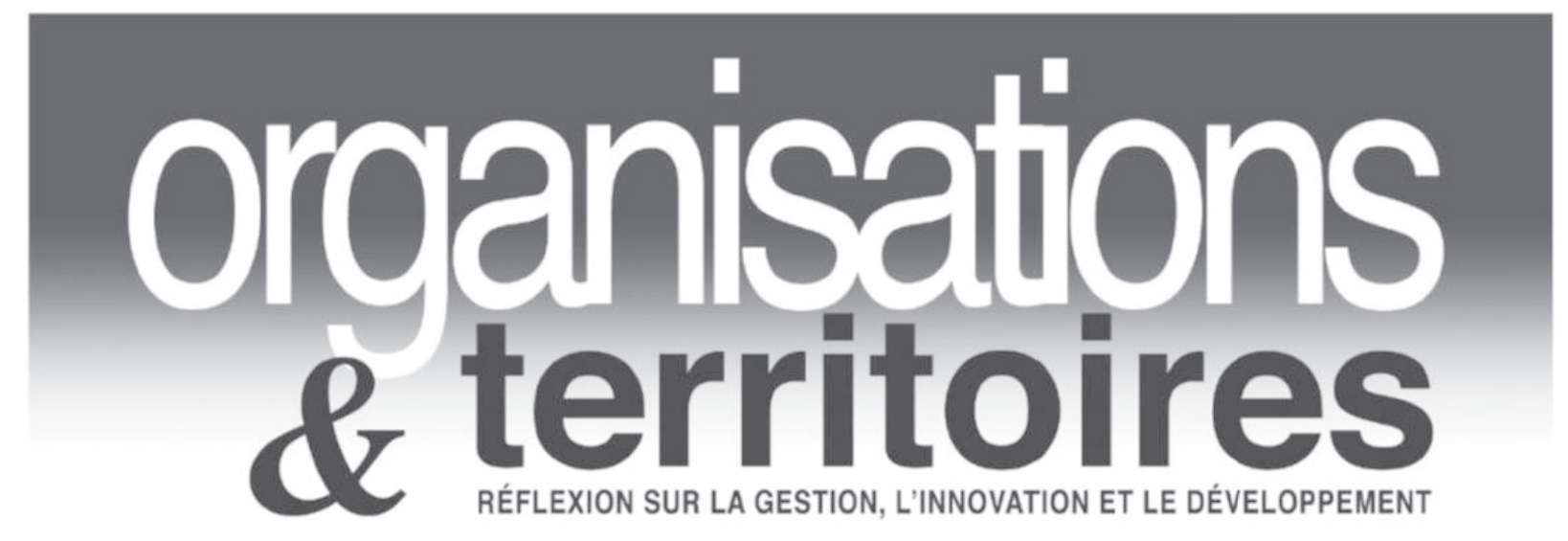

\section{Site Web}

\section{Www.uqac.ca/revuleot \\ Www.uqac.ca/revueot \\ www.uqac.ca/revueot \\ www.uqac.ca/revueot}

Nous vous invitons à l'explorer et à nous faire parvenir vos commentaires et suggestions afin d'en améliorer le contenu et la présentation. 